\title{
Robotic-Assisted Transanal Surgery for Rectal Schwannoma: A case report
}

\author{
Yan Jiun Huang ${ }^{1,2,6}$, Wei Lin Wang ${ }^{7}$ and Po Li Wei*1,2,3,4,5,7 \\ ${ }^{1}$ Department of Surgery, Taipei Medical University, Taiwan \\ ${ }^{2}$ Division of Colorectal Surgery, Department of Surgery, Taipei Medical University Hospital, Taiwan \\ ${ }^{3}$ Cancer Research Centre, Taipei Medical University Hospital, Taiwan \\ ${ }^{4}$ Department of Medical Research, Taipei Medical University Hospital, Taiwan \\ ${ }^{5}$ Graduate Institute of Cancer Biology and Drug Discovery, Taipei Medical University, Taiwan \\ ${ }^{6}$ The PhD Program for Translational Medicine, Taipei Medical University and Academia Sinica, Taiwan \\ ${ }^{7}$ Division of Colorectal Surgery, Department of Surgery, Wan Fang Hospital, Taiwan
}

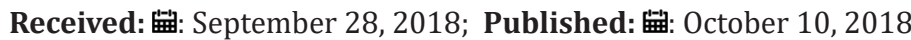

*Corresponding author: Po-Li Wei, Division of Colorectal Surgery, Department of Surgery, Taipei Medical University Hospital, 252

Wuxing Street, Sinyi District, Taipei 11031, Taiwan

\section{Abstract}

Aim: The aim of this stud was to present a case with uncommon rectal schwannoma undergoing robotic transanal surgery.

Background: Gastrointestinal schwannomas are uncommon and in particular, rectal schwannomas are even rarer. It is well established that schwannomas, nerve sheath tumors originating from Schwann cells, appear more frequently in the stomach and in the small intestine. Postoperative staining positive for S-100 protein and negative for smooth muscle markers, such as actin and desmin is essential for diagnosis. Traditional surgery requires resection of the rectum and patients are faced with longer hospital stay but robotic-assisted transanal approach enhanced faster recovery and shorter hospital stay.

Case Description: A 47-year-old female was found to have one rectal tumor during digital rectal examination performed as part of a health examination. Colonoscopy showed one submucosal mass with intact mucosa with hard consistency by forceps touching at $10 \mathrm{~cm}$ above anal verge. Transanal robotic-assisted tumor excision was done with final pathology showing rectal schwannoma.

Conclusion: Robotic-assisted transanal approach for local excision of rectal tumors with low risk of malignancy can be performed safely.

Keywords: Rectal schwannoma; Robotic surgery

\section{Background}

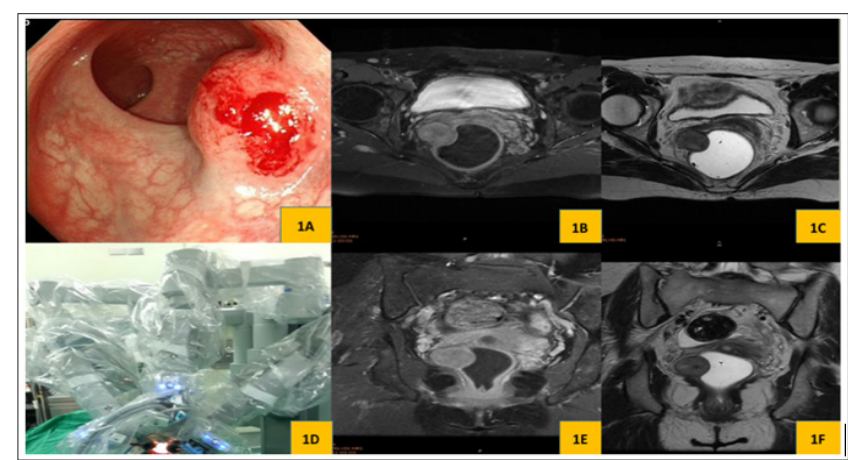

Figure 1.

A 47-year-old female was found to have one rectal tumor during digital rectal examination performed as part of a health examination and was admitted to hospital for complete work-up. She had no symptoms and her laboratory data were within the normal limits. Colonoscopy showed one submucosal mass with intact mucosa with hard consistency by forceps touching at $10 \mathrm{~cm}$ above anal verge (Figure 1A). Magnetic resonance imaging showed one nodular lesion ( $3.3 \times 3.0 \times 2.5 \mathrm{~cm}$ in size) at right aspect of lower rectum with characteristics of iso-signal intensity on T1W images (Figure 1B \& Figure1E), slight bright signal intensity on T2W images and heterogeneous enhancement (Figure 1C \& Figure 1F). There was no regional lymph node enlargement. Based on these findings, a tentative clinical diagnosis of Gastrointestinal Stromal Tumor (GIST) of rectum was made, and the patient underwent a robotic transanal tumor in toto excision (Figure 1D).

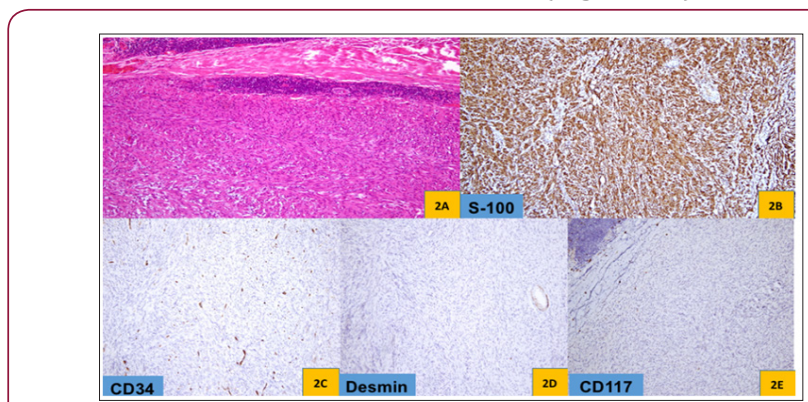

Figure 2. 
The patient was in jack-knife position after general anesthesia and Gelpoint path ${ }^{\circledR}$ was placed into anus. After pneumorectum at pressure of $10 \mathrm{mmHg}$ and da Vinci was docked for transanal minimal invasive surgery to remove the submucosal tumor and the wound was closed. Histologic examination revealed a submucosal nodular lesion composed of proliferative spindle tumor cells with plump wavy nuclei in short fascicles and thick collagen deposition (Figure 2A). Immunohistochemically, the tumor cells are diffusely positive for S100 protein (Figure 2B) and negative for CD34 (Figure 2C), desmin (Figure 2D) and CD117 (c-kit) (Figure 2E). A schwannoma is diagnosed pathologically. The resection margins were free of tumor cells. The patient made an uneventful recovery and was discharged 2 days after the operation.

\section{Discussion}

Schwannomas are nerve sheath tumors originating from Schwann cells. These lesions were first described by Herrera et al a group of Gastrointestinal Autonomic Nerve Tumors (GANTs) in 1984 [1]. Gastrointestinal schwannomas are uncommon and in particular, rectal schwannomas are rare [2]. In fact, it is well established that schwannomas appear more frequently in the stomach and in the small intestine [3]. In the current case, although preoperative tentative diagnosis of the submucosal tumor suggested a GIST, postoperative staining positive for S-100 protein, as in our case, and negative for smooth muscle markers, such as actin and desmin, which are positive in myogenic tumors is essential for differential diagnosis. Despite no report on malignant rectal schwannomas in the English literature, it is generally a benign tumor with a local recurrence of $30 \%$ after incomplete excision or $2 \%$ of distant metastasis [4].

\section{ISSN: 2574-1241}

DOI: 10.26717/BJSTR.2018.09.001871

Po Li Wei. Biomed J Sci \& Tech Res

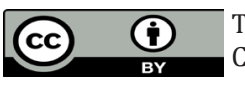

This work is licensed under Creative Commons Attribution 4.0 License

Submission Link: https://biomedres.us/submit-manuscript.php
We herein report a rare case of rectal schwannoma which was successfully treated by robotic transanal scarless surgery. This procedure is primarily used for local excision of selected benign and low-grade malignant tumor of the lower, middle and upper rectum via the natural orifice. Da Vinci $₫$ platform, is designed to overcome the shortcomings of traditional open and laparoscopic transanal surgery due to the increased range of motion, dexterity and most importantly the addition of second traction that is required for faster dissection, gain of adequate margin and depth in order to perform complete resection. Local excision using a robotic-assisted approach is a feasible surgical approach for benign rectal tumors such as small GIST and schwannoma.

\section{Conclusion}

Robotic-assisted transanal surgery is a feasible approach for local excision of rectal tumors with low risk of malignancy.

\section{References}

1. Herrera GA, Pinto de Moraes H, Grizzle WE, Han SG (1984) Malignant small bowel neoplasm of enteric plexus derivation (plexosarcoma). Light and electron microscopic study confirming the origin of the neoplasm. Dig Dis Sci 29: 275-284.

2. Maddalena Zippi, Roberta Pica, Renzo Scialpi, Claudio Cassieri, Eleonora Veronica Avallone, et al. (2013) Schwannoma of the rectum: A case report and literature review. World J Clin Cases 1(1): 49-51.

3. Wei Bing Wang, Wen Bin Chen, Jian Jiang Lin, Jia He Xu, Jin Hai Wang, et al. (2016) Schwannoma of the colon: A case report and review of the literature. Oncol Lett 11(4): 2580-2582.

4. Nassif MO, Trabulsi NH, Bullard Dunn KM, Nahal A, Meguerditchian AN (2013) Soft tissue tumors of the anorectum: Rare, complex and misunderstood. J Gastrointest Oncol 4: 82-94.

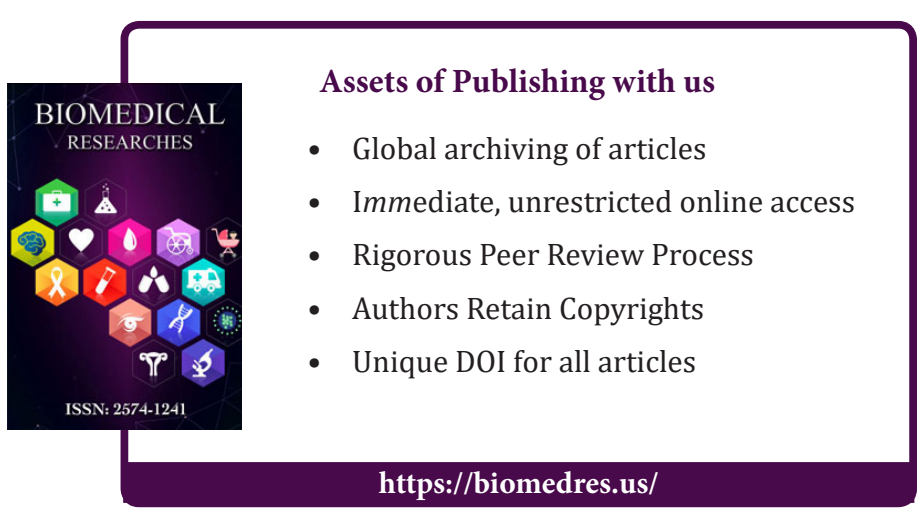

Cite this article: Yan Jiun H, Wei Lin W, Po Li Wei. Robotic-Assisted Transanal Surgery for Rectal Schwannoma: A case report. Biomed J 